\title{
Screening Mixtures of Small Molecules for Binding to Multiple Sites on the Surface of Tetanus Toxin C Fragment by Bioaffinity NMR
}

M. Cosman, L. Zeller, F. C. Lightstone, V. V. Krishnan, R. Balhorn

U.S. Department of Energy

\section{January 1, 2002}

Lawrence

Livermore

National

Laboratory 



\section{DISCLAIMER}

This document was prepared as an account of work sponsored by an agency of the United States Government. Neither the United States Government nor the University of California nor any of their employees, makes any warranty, express or implied, or assumes any legal liability or responsibility for the accuracy, completeness, or usefulness of any information, apparatus, product, or process disclosed, or represents that its use would not infringe privately owned rights. Reference herein to any specific commercial product, process, or service by trade name, trademark, manufacturer, or otherwise, does not necessarily constitute or imply its endorsement, recommendation, or favoring by the United States Government or the University of California. The views and opinions of authors expressed herein do not necessarily state or reflect those of the United States Government or the University of California, and shall not be used for advertising or product endorsement purposes.

This work was performed under the auspices of the U. S. Department of Energy by the University of California, Lawrence Livermore National Laboratory under Contract No. W-7405-Eng-48.

This report has been reproduced directly from the best available copy.

Available electronically at http://www.doc.gov/bridge

Available for a processing fee to U.S. Department of Energy

And its contractors in paper from

U.S. Department of Energy

Office of Scientific and Technical Information

P.O. Box 62

Oak Ridge, TN 37831-0062

Telephone: (865) 576-8401

Facsimile: (865) 576-5728

E-mail: reports@adonis.osti.gov

Available for the sale to the public from

U.S. Department of Commerce

National Technical Information Service

5285 Port Royal Road

Springfield, VA 22161

Telephone: (800) 553-6847

Facsimile: (703) 605-6900

E-mail: orders@ntis.fedworld.gov

Online ordering: http://www.ntis.gov/ordering.htm

OR

Lawrence Livermore National Laboratory

Technical Information Department's Digital Library

http://www.llnl.gov/tid/Library.html 



\section{Screening Mixtures of Small Molecules for Binding to Multiple Sites on the Surface}

\section{of Tetanus Toxin C Fragment by Bioaffinity NMR}

Monique Cosman ${ }^{1 *}$, Loreen Zeller ${ }^{2}$, Felice C. Lightstone ${ }^{1}$, V. V. Krishnan ${ }^{1}$, and Rod Balhorn $^{1 *}$

${ }^{1}$ Biology and Biotechnology Research Program and ${ }^{2}$ Chemistry and Materials Science Directorate, Lawrence Livermore National Laboratory, Livermore, California 94551.

*To whom correspondence and reprint requests should be addressed, MC: phone, (925) 423-1647; fax, (925) 424-3120; e-mail cosman1@llnl.gov. RB: phone, (925) 423-1647; fax, (925) 424-3120; e-mail balhorn2@llnl.gov. 


\section{Abbreviations:}

TeNT, tetanus neurotoxin; TetC. Tetanus toxin C fragment; BoNT, botulinum neurotoxin; ACD, Available Chemicals Directory; ESI-MS, electrospray ionization mass spectroscopy; NMR, nuclear magnetic resonance; trNOE, transfer nuclear Overhauser effect; SIA, N-acetylneuraminic acid (sialic acid); DOX, doxorubicin hydrochloride; SAR-RGDSP, Sarcosine-Arg-Gly-Asp-Ser-Pro; SQNYPIV, Ser-Gln-Asn-Tyr-Pro-IleVal, NMP-biocytin, 3-(N-maleimidopropionyl)biocytin, NF-GalPyr, naphthofluoresceindi- $\beta$-galactopyranoside; NGA, N-acetyl-galactosamine, LAC, lactose; GAL, galactose; GLU, glucose.. 


\section{Abstract}

The clostridial neurotoxins include the closely related tetanus (TeNT) and botulinum (BoNT) toxins. Botulinum toxin is used to treat severe muscle disorders and as a cosmetic wrinkle reducer. Large quantities of botulinum toxin have also been produced by terrorists for use as a biological weapon. Because there are no known antidotes for these toxins, they thus pose a potential threat to human health whether by an accidental overdose or by a hostile deployment. Thus, the discovery of high specificity and affinity compounds that can inhibit their binding to neural cells can be used as antidotes or in the design of chemical detectors. Using the crystal structure of the $\mathrm{C}$ fragment of the tetanus toxin (TetC), which is the cell recognition and cell surface binding domain, and the computational program DOCK, sets of small molecules have been predicted to bind to two different sites located on the surface of this protein. While Site- 1 is common to the TeNT and BoNTs, Site-2 is unique to TeNT. Pairs of these molecules from each site can then be linked together synthetically to thereby increase the specificity and affinity for this toxin. Electrospray ionization mass spectroscopy was used to experimentally screen each compound for binding. Mixtures containing binders were further screened for activity under biologically relevant conditions using nuclear magnetic resonance (NMR) methods. The screening of mixtures of compounds offers increased efficiency and throughput as compared to testing single compounds and can also evaluate how possible structural changes induced by the binding of one ligand can influence the binding of the second ligand. In addition, competitive binding experiments with mixtures containing ligands predicted to bind the same site could identify the best binder for that site. NMR transfer nuclear Overhauser effect (trNOE) confirm that TetC binds doxorubicin but that 
this molecule is displaced by $\mathrm{N}$-acetylneuraminic acid (sialic acid) in a mixture that also contains 3-sialyllactose (another predicted site 1 binder) and bisbenzimide 33342 (nonbinder). A series of five predicted Site-2 binders were then screened sequentially in the presence of the Site-1 binder doxorubicin. These experiments showed that the compounds lavendustin A and naphthofluorescein-di-( $\beta$-D-galactopyranoside $)$ binds along with doxorubicin to TetC. Further experiments indicate that doxorubicin and lavendustin are potential candidates to use in preparing a bidendate inhibitor specific for TetC. The simultaneous binding of two different predicted Site-2 ligands to TetC suggests that they may bind multiple sites. Another possibility is that the conformations of the binding sites are dynamic and can bind multiple diverse ligands at a single site depending on the pre-existing conformation of the protein, especially when doxorubicin is already bound. 


\section{Introduction}

The structurally and functionally related tetanus toxin (TeNT) and the botulinum toxins (BoNTs) are members of the family of clostridial neurotoxins. Both selectively concentrate at the synapse of axons in vertebrate motor neurons and are the most potent toxins known to man (1). The recent interest in clostridial neurotoxins arises from both the increased frequency of their use in medicine and the potential threat that they might be used by terrorist groups or other nations as biological weapons $(2,3)$. The entry of these toxins into neuronal cells proceeds through the initial binding of the toxin to gangliosides on the cell surface. Thus effective inhibitors of binding to neuron cells can be developed for use as antidotes or serve as molecular recognition materials for affinitybased chemical sensors in detecting and identifying when these toxins have been deployed.

The Clostridium tetani and Clostridium botulinum bacteria synthesize teNT and BoNTs, respectively, as single $150 \mathrm{kDa}$ polypeptides that are subsequently clipped into two chains held together by a single disulfide bond. The toxins enter the neuron through processes involving specific recognition, endocytosis and intracellular transport $(l)$. TeNT targets inhibitory neurons within the central nervous system spinal cord causing a spastic paralysis, while BoNTs target peripheral sensory neurons resulting in flaccid paralysis (4). The heavy chain binds specifically to presynaptic neuronal cells, presumably to gangliosides located on the surface of the cell (I). After the entire toxin is internalized into the cells via vesicles, the light chain is translocated into the cytosol and inhibits neurotransmitter release by targeting and cleaving one of three proteins, VAPM, SNAP-25 or syntaxin (5-7). 
Tetanus neurotoxin (TeNT) has been shown to specifically bind gangliosides of the Glb series, GD1b or GT1b (8-11). The receptor binding subunit of TeNT has been shown to be a $50 \mathrm{kDa}$ polypeptide comprising the C-terminal 452 amino acids of the heavy chain $\left(\mathrm{H}_{\mathrm{c}}\right)$, more commonly termed the $\mathrm{C}$ fragment $(12,13)$. In particular, the last 34 residues of the $\mathrm{C}$ fragment participate in ganglioside recognition, with the residue His 1293 identified as being critical for binding $(13,14)$. Several crystal structures of the tetanus toxin $\mathrm{C}$ fragment (TetC) (e.g. pdb codes: $1 \mathrm{AF9}$ and 1A8D, $(15,16)$ and residues 875 to 1315 ) have been reported in the protein databank (http://www.rcsb.org/pdb/). These structures include complexes with the individual ganglioside sugar components $\mathrm{N}$ acetyl-galactosamine (NGA, pdb code: 1D0H), galactose (GAL, pdb code: 1DIW), lactose (LAC, pdb code: 1DLL), and sialic acid (SIA, pdb code: 1DFQ) (4)), and with a ganglioside GT1B derivative (pdb code: 1FV2, (17). The TetC structure can be divided into two subdomains, a lentil lectin-like $\mathrm{N}$-terminal jelly roll domain and a $\mathrm{C}$-terminal $\beta$ trefoil domain (18) (Fig. 1). The sugars individually bind to the surface of TetC in four different sites, which are widely spaced along one edge of the $C$-terminal $\beta$-trefoil subdomain (4). In contrast, the derivative of the ganglioside GT1b, which is composed of glucose-GAL-NGA-GAL- $\alpha$ SIA- $\beta$ SIA- $\alpha$ SIA, binds to same site as does lactose ( 4 , 17). The antitumor drug doxorubicin was also predicted by computer docking methods to bind in the same site, designated as Site-1 in this study (Fig. 2) (19). Site-1 is a common surface feature found in the structures of both $\mathrm{TetC}$ and BoNT, and a recent crystal structure of BoNT/B in complex with doxorubicin (20) is in agreement with doxorubicin binding in Site-1. In addition, crystal structures of TetC in complex with doxorubicin or 
sialic acid that are currently in progress also show that these two compounds bind to Site1 (S. Swaminathan, personal communication).

In the current study, computational docking methods were used to identify the potential binding site on the surface of TetC that would be unique to this toxin, designated as Site-2. Linking two ligands from each binding site to form a bidendate ligand would be expected to increase the specificity and affinity for TetC (2I). The resulting bidendate ligand can be used to develop a sensitive molecular sensor to differentiate between the TeNT and BoNT toxins. We used computational docking routines to identify a small subset of ligands found in the Available Chemical Database (ACD) that are predicted to bind to Site-2. These ligands were then checked experimentally for binding activity by using electrospray ionization mass spectroscopy (ESI-MS). However, a potential problem with many biological systems is that the activity observed can be the result of the sum total of weak, nonspecific binding of several mixture components in the assay. In addition, the binding of one ligand at Site-1 may change the conformation of the protein and affect the subsequent binding of ligands at Site-2. Moreover, the ligands identified from Site 2 may be incompatible with those identified as binding in Site 1 under similar solvent conditions, making it impractical to link them together to produce a bidendate ligand. Thus mixtures containing Site- 1 and Site-2 ligands were further screened for binding to TetC by using nuclear magnetic resonance (NMR) spectroscopy. The selective detection of transfer nuclear Overhauser effects (trNOEs) makes use of the observation that small molecules (relative molecular mass up to about $2 \mathrm{kDa}$ ) exhibit strong negative trNOEs when bound to receptor proteins. and can thus be differentiated from nonbinding molecules with weak positive NOEs (22). 
The results for the NMR screening for binding to TetC of mixtures containing combinations of three Site- 1 and five Site-2 ligands are reported here.

\section{Materials and Methods}

Recombinant Protein, Ligands, and Other Reagents

A recombinant form of TetC was purchased from Roche Molecular Biochemicals (Indianapolis, IN). The ligands purchased from Sigma-Aldrich Chemical Co. (St. Louis, MO) were N-acetylneuraminic acid (sialic acid), 3'-sialyllactose, Sarcosine-Arg-GlyAsp- Ser-Pro, 3-(N-maleimidopropionyl)biocytin, lavendustin A, naphthofluorescein-di$\beta$-galactopyranoside. The following compounds were purchased from CalbiochemNovabiochem Corp (La Jolla, CA): doxorubicin hydrochloride, bisbenzimide H33342, Ser-Gln-Asn-Tyr-Pro-Ile-Val and N-acetyl neuramic acid (sialic acid). Deuterated DMSO and D2O were purchased from Isotech . (St. Louis, MO).

\section{Computer Modeling Methods}

Coordinates obtained from the crystal structure of TetC ( $\mathrm{pdb}$ code 1A8D) were used for the modeling and docking studies. Four binding sites, including Site-1 and Site 2 , on the surface of TetC were identified by calculating the solvent accessible surface area (SASA) using the SPHGEN routine from DOCK (23), which packs clusters of spheres into structural pockets. The DOCK program (24-26) computationally screens the Available Chemical Database (ACD, version 97.2) of more than 200,000 commercially available compounds and predicts which molecules will likely bind tightly to the target 
receptor. A total of 127,475 compounds were included in the final version of the screening database; these were sorted into five separate lists for each value of total charge. In order to focus the study within the size and charge ranges expected for lead drug candidates, compounds with an absolute charge $>3$, as determined by using Gasteiger Marsili charges (27) in Sybyl (Tripos, Inc., St. Louis, MO), and with $<10$ or $>$ 80 heavy atoms were removed. The procedure used to computationally dock each compound has been previously described in detail by Lightstone et al. (19). Briefly, different orientations of each ligand within a site were scored for best intermolecular van der Waals and electrostatic force field potentials using AMBER (28) and for contact scoring (29), which is based on a simplified Lennard-Jones function. The ligand was held in a defined structure, thus possible conformational changes in either the ligand or the protein were not taken in account. Screening different conformers of each ligand would have required a tremendous increase in computational time and would have been contrary to our intention of screening as large a number of small molecules as quickly as possible with very high throughput. Separate lists of the top $1 \%$ ligands obtained by each scoring method (approximately 1000 compounds) were divided according to charge and examined further in more detail, one at a time. A new sphere-atom matching scheme in DOCK (23) was used to visually inspect likely candidates using computer graphics. The DOCK runs were performed on Silicon Graphics workstations with multiple $\mathrm{R} 8000 / \mathrm{R} 10000$ processors, and the overall run time ranged from 800 to $1350 \mathrm{CPU}$ hours per binding site. 
Electrospray Ionization Mass Spectroscopy (ESI-MS)

ESI-MS was used to experimentally determined which of the computationallydetermined candidates bind or do not bind to $\mathrm{Tet} C$. Aliquots of TetC were hydrolyzed in $\mathrm{HCl}$ and the amount of protein present determined by quantitative amino acid analysis (Structural Protein Laboratory, University of California, Davis, CA). Both TetC and ligands were dissolved in $3 \mathrm{mM}$ ammonium acetate, $\mathrm{pH} 7.6 \mathrm{buffer} / 12 \% \mathrm{v} / \mathrm{v}$ methanol.

Twenty microliter samples in which the protein concentration $(3-13 \mu \mathrm{M})$ was kept constant and the tetC:ligand ratio was varied from ?? to ?? in ?? steps were analyzed by ESI-MS on a Mariner orthogonal acceleration time-of-flight instrument (PE Biosystems, Framingham, M) within 20 minutes of mixing. The sample infusion rate was $1 \mu \mathrm{l} /$ minute through 60 and $25 \mu \mathrm{m}$ id capillaries. The spectra were acquired at room temperature and summed over 25 scans. The ion intensities were normalized to the protein peak [(bound protein)/(unbound protein)]. The multiply charged spectra were deconvoluted with the Biospec Data Explorer software (PE Biosystems). The slope from a plot of (bound protein)/(unbound protein) versus $1 /$ [ligand] was used to obtain a dissociation constant for the ligand (30). Instrument settings, such as gas flow rates, the number of scans, and declustering potentials were optimized and kept constant for each set of experiments corresponding to a specific complex. A range of declustering voltages were tried since mild settings were necessary to probe noncovalent interactions. The declustering potential that a complex can withstand without dissociating can provide a qualitative estimate of the strength and nature of the noncovalent interaction. 
NMR transfer nuclear Overhauser effect spectroscopy (trNOESY) experiments

Freeze-dried samples for NMR measurements were dissolved in $100 \% \mathrm{D}_{2} \mathrm{O}$.

Studies on TetC and doxorubicin dissolved in $5 \mathrm{mM}$ sodium phosphate, $\mathrm{pH} 7.0$ buffer were initially carried out. Since no significant changes were observed in the spectra when the buffer was not included and several of the ligands precipitated when buffer was present, subsequent experiments were carried out in $\mathrm{D}_{2} \mathrm{O}$ only. Some of the ligands (biocytin, lavendustin, and galactopyranoside) precipitated in buffer or water, and were thus first dissolved in a small volume $(100 \mu \mathrm{l})$ of deuterated DMSO before adding an aliquot $(\sim 20 \mu \mathrm{l})$ to TetC in $\mathrm{D}_{2} \mathrm{O}$. The final concentration of DMSO in total volume of $\mathrm{D}_{2} \mathrm{O}$ in the mixture used for the NMR experiments was $<7.6 \%$. Three milligrams of TetC were dissolved in $1.0 \mathrm{ml}$ of $\mathrm{D}_{2} \mathrm{O}$ and centrifuged at top speed for 5 minutes to remove insoluble material. The TetC concentrations used in the NMR experiments were $\sim 20$ to $60 \mu \mathrm{M}$, while the molar ratio of TetC:doxyrubicin was varied from a range of $1: 10$ to 1:50 in order to determine a good, consistent value to use in studying the mixtures. A final molar ratio of TetC:ligand of 1:30 was chosen based on the best sensitivity observed in the NMR spectra for all the ligands at this ratio.

All spectra were measured on Varian INOVA $600 \mathrm{MHz}$ spectrometer at either 20 ${ }^{\circ} \mathrm{C}$ or $30^{\circ} \mathrm{C}$, as stated in the figure captions. The phase sensitive 2D-NOESY experiments of ligands or mixtures of ligands were carried out at $900 \mathrm{~ms}$ mixing times, while mixtures of ligands in the presence of TetC were carried out at 200 and $300 \mathrm{~ms}$ mixing times. The spectral width was $20 \mathrm{ppm}$ in both dimensions with 600 increments in $\mathrm{t}_{1}$ (TPPI) each with 64 scans of 2048 data points in $t_{2}$. Prior to Fourier transformation, the data matrix was zero filled to 2048 by 2048 data points. 


\section{Results and Discussion}

\section{Computational Docking}

The solvent accessible surface of TetC was calculated to identify surface pockets as potential sites for ligand binding. The volumes of the pockets were calculated by filling them with a combination of spheres with different radii. Fifty-two pockets were identified as potential binding sites for small molecules. Four of these were selected for further study because of proximity to the ganglioside binding site (17) or because of the uniqueness of the site to TetC. Previously, we reported the results of our computational docking identification of Site- 1 and a set of potential binders (19). Site-1 is a common surface feature in the structures of both TeNT and BoNTs and is the binding site for a ganglioside GT1b derivative (17). The small molecules identified by screening the Available Chemicals Directory (ACD) and tested positive for binding by ESI-MS include the antitumor drug doxorubicin, which was found to bind to $\mathrm{TetC}$ with an appreciable binding constant of 9.4 uM (as determined using a GT lb liposome assay) (19). Other predicted Site-1 binders that were confirmed to bind by mass spectroscopy were $D-(+)-$ cellotetraose, neohesperidin, the peptide Gly-Arg-Gly-Asp-Ser, and hemorphin-5:

In the current study, the results are reported for the second of the four sites. Site 2 is a deep surface pocket unique to TetC that is located in-between the C-terminal $\beta$-trefoil and N-terminal jelly-roll subdomains. It is proximal to Site-1, making it attractive for possible linking of two ligands, one from each site. The ACD was screened for ligands that could bind to this site, and ranked by energy and contact scores. The $1 \%$ of scored compounds were visually examined qualitatively for specific interactions, such as charge 
and van der Waals forces. A variety of molecules were chosen to represent the spectrum of available compounds even though peptides dominated. Twenty-nine final compounds were selected as potential binders. One of the ligands, lavendustin A, is shown docked into Site-2 in Figure 2D. Due to their limited availability, only eleven of these ligands were purchased and tested for binding.

\section{Electrospray Ionization Mass Spectroscopy}

The mass spectrometry results obtained for all the ligands that were tested are summarized in Table 1

Table 1. Ligands tested by ESI-MS for noncovalent complex formation with TetC Site-2

\begin{tabular}{|l|l|}
\hline complexes observed & no complexes observed \\
\hline Sar-Arg-Gly-Asp-Ser-Pro & \\
\hline Ser-Gln-Asn-Tyr-Pro-Ile-Val & \\
\hline 3-(N-Maleimidopropionyl)biocytin & \\
\hline Lavendustin A & \\
\hline Naphthofluorescein & \\
\hline & \\
\hline
\end{tabular}

Ligand binding, as defined in these experiments, occurs when a new mass peak appears at the expected mass $/$ charge $(\mathrm{m} / \mathrm{z})$ ratio for the complex. For example, an ESI spectrum of TetC and the TetC-lavendustin A complex is shown in Figure 3. Only those complexes that are stable to ionization and electrospray are observed. The main concern when using 
ESI-MS to investigate solution phase characteristics is the induction of complex formation via nonspecific aggregation or gas phase-induced binding. These contributions may arise from either the electrospray process or the solution conditions. To minimize this problem, low ratios of ligand concentration were used. To determine the concentration range, a series of saturation binding curves were generated for each molecule. Complexes were prepared by adding increasing amounts of ligand while keeping the TetC concentration constant until saturation was achieved. The ratio of bound protein to unbound protein is linearly correlated to the ligand concentration (the inverse was plotted for dissociation constant determination, as stated in Materials and Methods) up to the saturation point, beyond which increasing the ligand concentration will not produce any more bound protein. Thus, the ligand concentration was kept within the linear region of the saturation binding curves for all binding experiments.

Complexes stabilized by weak binding forces may be labile in the gas phase. To minimize dissociation of the complex, all the samples were electrosprayed at room temperature. The declustering potential was kept between 50 and $200 \mathrm{~V}$ to help minimize complex dissociation. All but one of the ligand complexes that were tested withstood the high declustering voltages in the gas phase, indicating that the complexes were primarily stabilized by electrostatic interactions. Complexes which required an applied nozzle potential of less than or equal to $100 \mathrm{~V}$ indicated that the interactions were hydrophobic in nature.

These results show that ESI-MS is a reasonably efficient screening method for confirming ligand binding. 
Competitive binding experiments by NMR transfer NOE (trNOE) experiments.

Binding studies of mixtures of compounds were also examined for binding to TetC under biologically relevant conditions using NMR. A strong positive NOESY (nuclear Overhauser effect spectroscopy) crosspeak is observed for binders, as opposed to weakly negative or zero NOE crosspeaks for the same mixture of compounds in the absence of TetC (Fig. 6). Thus the sign flip of the NOE cross peak in a trNOESY spectrum between the free versus bound states acts as a simple binary filter to distinguish binders from nonbinders. However, the sign flip was not always consistent for all ligands. Some ligands exhibited resonances that had positive sign with and without the presence of TetC. However, the intensity of the same sign peaks were always much stronger in the presence of $\mathrm{TetC}$ when bound and very weak when protein was absent. Thus, both the sign and intensity of the crosspeaks were taken into account to distinguish binders from nonbinders.

The results show that the trNOESY method is well suited for the identification of binding activity. Several advantages of the method are: 1) Separation of the components of a mixture prior to activity tests is not necessary. 2) Small amounts of protein are sufficient since the method is ligand-based and the ligand is added in a 5-50-fold excess. 3) The protein can be recovered after dialysis and used for further tests. 4) Neither the protein nor the ligand must be immobilized. 5) The procedure can be used in competitive assays and determine if two or more compounds bind simultaneously. 6) Binding studies can be carried out under biologically relevant conditions. 


\section{Acknowledgements}

We gratefully acknowledge the support by the Department of Energy's Chemical and Biological Non-Proliferation Program to RB and the Lawrence Livermore National Laboratory Exploratory LDRD grant 01-ERD-046 to MC. This work was performed under the auspices of the of the U.S. Department of Energy by Lawrence Livermore National Laboratory under Contract W-7405-Eng-48. 


\section{References}

1. Montecucco, C., and Schiavo, G. (1995) Structure and Function of Tetanus and Botulinum Neurotoxins Quarterly Reviews of Biophysics 28, 423-472.

2. Henderson, D. A. (2000) Bioterrorism Int J Clin Pract Suppl, 32-6.

3. Arnon, S. S., Schechter, R., Inglesby, T. V., Henderson, D. A., Bartlett, J. G., Ascher, M. S., Eitzen, E., Fine, A. D., Hauer, J., Layton, M., Lillibridge, S., Osterholm, M. T., O'Toole, T., Parker, G., Perl, T. M., Russell, P. K., Swerdlow, D. L., and Tonat, K. (2001) Botulinum toxin as a biological weapon: medical and public health management Jama 285, 1059-70.

4. Emsley, P., Fotinou, C., Black, I., Fairweather, N. F., Charles, I. G., Watts, C., Hewitt, E., and Isaacs, N. W. (2000) The structures of the H(C) fragment of tetanus toxin with carbohydrate subunit complexes provide insight into ganglioside binding $J$ Biol Chem 275, 8889-94.

5. Schiavo, G., Benfenati, F., Poulain, B., Rossetto, O., Delaureto, P. P., Dasgupta, B. R., and Montecucco, C. (1992) Tetanus and Botulinum-B Neurotoxins Block Neurotransmitter Release By Proteolytic Cleavage of Synaptobrevin Nature 359, 832-835.

6. Blasi, J., Chapman, E. R., Yamasaki, S., Binz, T., Niemann, H., and Jahn, R. (1993) Botulinum Neurotoxin-Cl Blocks Neurotransmitter Release By Means of Cleaving Hpc-1/Syntaxin Embo Journal 12, 4821-4828.

7. Blasi, J., Chapman, E. R., Link, E., Binz, T., Yamasaki, S., Decamilli, P., Sudhof, T. C., Niemann, H., and Jahn, R. (1993) Botulinum Neurotoxin-a Selectively Cleaves the Synaptic Protein Snap-25 Nature 365, 160-163. 
8. Morris, N. P., Consiglio, E., Kohn, L. D., Habig, W. H., Hardegree, M. C., and Helting, T. B. (1980) Interaction of fragments B and C of tetanus toxin with neural and thyroid membranes and with gangliosides $J$ Biol Chem 255, 6071-6.

9. Holmgren, J., Elwing, H., Fredman, P., and Svennerholm, L. (1980) Polystyreneadsorbed gangliosides for investigation of the structure of the tetanus-toxin receptor Eur J Biochem 106, 371-9.

10. Rogers, T. B., and Snyder, S. H. (1981) High affinity binding of tetanus toxin to mammalian brain membranes $J$ Biol Chem 256, 2402-7.

11. Winter, A., Ulrich, W. P., Wetterich, F., Weller, U., and Galla, H. J. (1996) Gangliosides in phospholipid bilayer membranes: interaction with tetanus toxin Chem Phys Lipids 81, 21-34.

12. Helting, T. B., and Zwisler, O. (1977) Structure of tetanus toxin. I. Breakdown of the toxin molecule and discrimination between polypeptide fragments $J$ Biol Chem 252, 187-93.

13. Halpern, J. L., and Loftus, A. (1993) Characterization of the receptor-binding domain of tetanus toxin $J$ Biol Chem 268, 11188-92.

14. Shapiro, R. E., Specht, C. D., Collins, B. E., Woods, A. S., Cotter, R. J., and Schnaar, R. L. (1997) Identification of a ganglioside recognition domain of tetanus toxin using a novel ganglioside photoaffinity ligand $J$ Biol Chem 272, 30380-6.

15. Knapp, M., Segelke, B., and B., R. (1998) The 1.61 Angstrom structure of the tetanus Toxin ganglioside binding region solved by MAD and MIR phase combination. Am. Crystallogr. Assoc. 25, 90. 
16. Umland, T. C., Wingert, L. M.. Swaminathan, S., Furey, W. F., Schmidt, J. J., and Sax, M. (1997) Structure of the receptor binding fragment HC of tetanus neurotoxin Nat Struct Biol 4, 788-92.

17. Fotinou, C., Emsley, P., Black, I., Ando, H., Ishida, H., Kiso, M., Sinha, K. A., Fairweather, N. F., and Isaacs, N. W. (2001) The crystal structure of tetanus toxin Hc fragment complexed with a synthetic GT1b analogue suggests cross-linking between ganglioside receptors and the toxin Journal of Biological Chemistry 276. 32274-32281.

18. Umland, T. C., Wingert, L. M., Swaminathan, S., Furey, W. F., Schmidt, J. J., and Sax, M. (1997) Structure of the receptor binding fragment HC of tetanus neurotoxin [letter] Nature Structural Biology 4, 788-92.

19. Lightstone, F. C., Prieto, M. C., Singh, A. K., Piqueras, M. C., Whittal, R. M., Knapp, M. S., Balhorn, R., and Roe, D. C. (2000) Identification of novel small molecule ligands that bind to tetanus toxin Chemical Research in Toxicology 13 , 356-62.

20. Eswaramoorthy, S., Kumaran, D., and Swaminathan, S. (2001) Crystallographic evidence for doxorubicin binding to the receptor-binding site in Clostridium botulinum neurotoxin B Acta Crystallogr D Biol Crystallogr 57, 1743-6.

21. Hajduk, P. J., Meadows, R. P., and Fesik, S. W. (1997) Discovering high-affinity ligands for proteins Science 278, 497,499.

22. Henrichsen, D., Ernst, B., Magnani, J. L., Wang, W. T., Meyer, B., and Peters, T. (1999) Bioaffinity NMR spectroscopy: Identification of an E-selectin antagonist 
in a substance mixture by transfer NOE Angewandte Chemie-International Edition 38, 98-102.

23. Ewing, T. J. A., and Kuntz, I. D. (1997) Critical evaluation of search algorithms for automated molecular docking and database screening Journal of Computational Chemistry 18, 1175-1189.

24. DesJarlais, R. L., Sheridan, R. P., Seibel, G. L., Dixon, J. S., Kuntz, I. D., and Venkataraghavan, R. (1988) Using shape complementarity as an initial screen in designing ligands for a receptor binding site of known three-dimensional structure J Med Chem 31, 722-9.

25. Kuntz, I. D., Blaney, J. M., Oatley, S. J., Langridge, R., and Ferrin, T. E. (1982) A geometric approach to macromolecule-ligand interactions $J$ Mol Biol 161, 26988.

26. Meng, E. C., Shoichet, B. K., and Kuntz, I. D. (1992) Automated Docking With Grid-Based Energy Evaluation Journal of Computational Chemistry 13, 505-524.

27. Gasteiger, J., Pfortner, M., Sitzmann, M., Hollering, R., Sacher, O., Kostka, T., and Karg, N. (2000) Computer-assisted synthesis and reaction planning in combinatorial chemistry Perspectives in Drug Discovery and Design 20, 245-264.

28. Kollman, P. A., Weiner, S., Seibel, G., Lybrand, T., Singh, U. C., Caldwell, J., and Rao, S. N. (1986) Modeling complex molecular interactions involving proteins and DNA Ann NY Acad Sci 482, 234-44.

29. DesJarlais, R. L., Sheridan, R. P., Dixon, J. S., Kuntz, I. D., and Venkataraghavan, R. (1986) Docking flexible ligands to macromolecular receptors by molecular shape $J$ Med Chem 29, 2149-53. 
30. Greig, M. J., Gaus, H., Cummins, L. L., Sasmor, H., and Griffey, R. H. (1995)

Measurement of Macromolecular Binding Using Electrospray Mass Spectrometry

- Determination of Dissociation Constants For Oligonucleotide-Serum Albumin

Complexes Journal of the American Chemical Society 117, 10765-10766. 


\section{Figure Captions:}

Figure 1: The overall fold of the tetanus toxin $\mathrm{C}$ fragment in the high resolution crystal structure.

Figure 2: Views of TetC in complex with (A) sialic acid (yellow), NGA (red), galactose (blue) and lactose (green) from the crystal structure (Emsley et.al. (2000) JBC 12, 889-8894); (B) ganglioside GTB derivative from the crystal structure (Foutinou et. al. (2001) JBC 276, 32274-32281; (C) doxorubicin from computational docking prediction (Site-1); and (D) lavendustin from computational docking prediction (Site-2).

Figure 3: Site-1 ligand structures and molecular weights.

Figure 4: Site-2 ligands structures and molecular weights.

Figure 5: Mass Spectrum of TetC at $100 \mathrm{~V}$. The molecular mass is 51,809.6 Da (b) and (c). The mass spectrum of the complex of lavendustin and $\mathrm{TetC}$ (b) at a declustering voltage of $100 \mathrm{~V}$ showing ligand binding and (c) at a declustering voltage of $200 \mathrm{~V}$ shows no binding.

Figure 6: Left: 2D NOESY spectrum at 900 ms mixing time of the compound mixture composed of doxorubicin, lavendustin, and biocytin in $\mathrm{D}_{2} \mathrm{O}$ at $30^{\circ} \mathrm{C}$. The NOEs are weak and negative for most resonances. Right: 2D transfer NOESY at $300 \mathrm{~ms}$ mixing time of the same mixture in the presence of TetC show strong positive crosspeaks for doxorubicin and lavendustin, indicating that these two ligands bind, while biocytin does not.

Figure 7: 2D transfer NOESY spectra at $200 \mathrm{~ms}$ mixing time and $20{ }^{\circ} \mathrm{C}$ showing binding of doxorubicin (leftmost panel, Site-1 binder) and a series of predicted Site-2 
binders added sequentially. The presence of new crosspeaks (boxed) indicates binding. Both lavendustin binds and appears to be partially displaced by galactopyranoside, suggesting that these two interact in the same binding site 


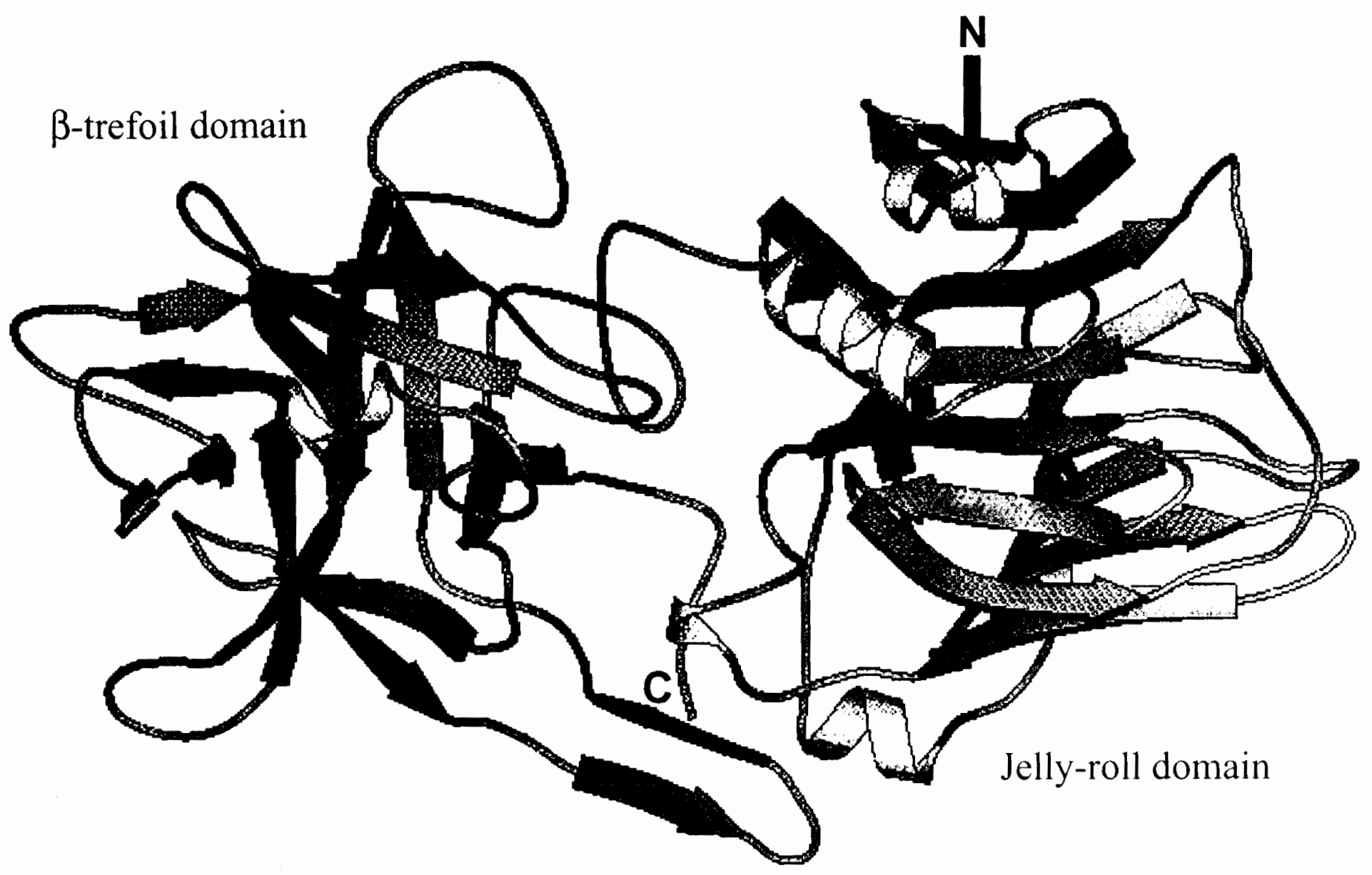

Figure 1: The overall fold of the the tetanus toxin $\mathrm{C}$ fragment in the high resolution crystal structure. 


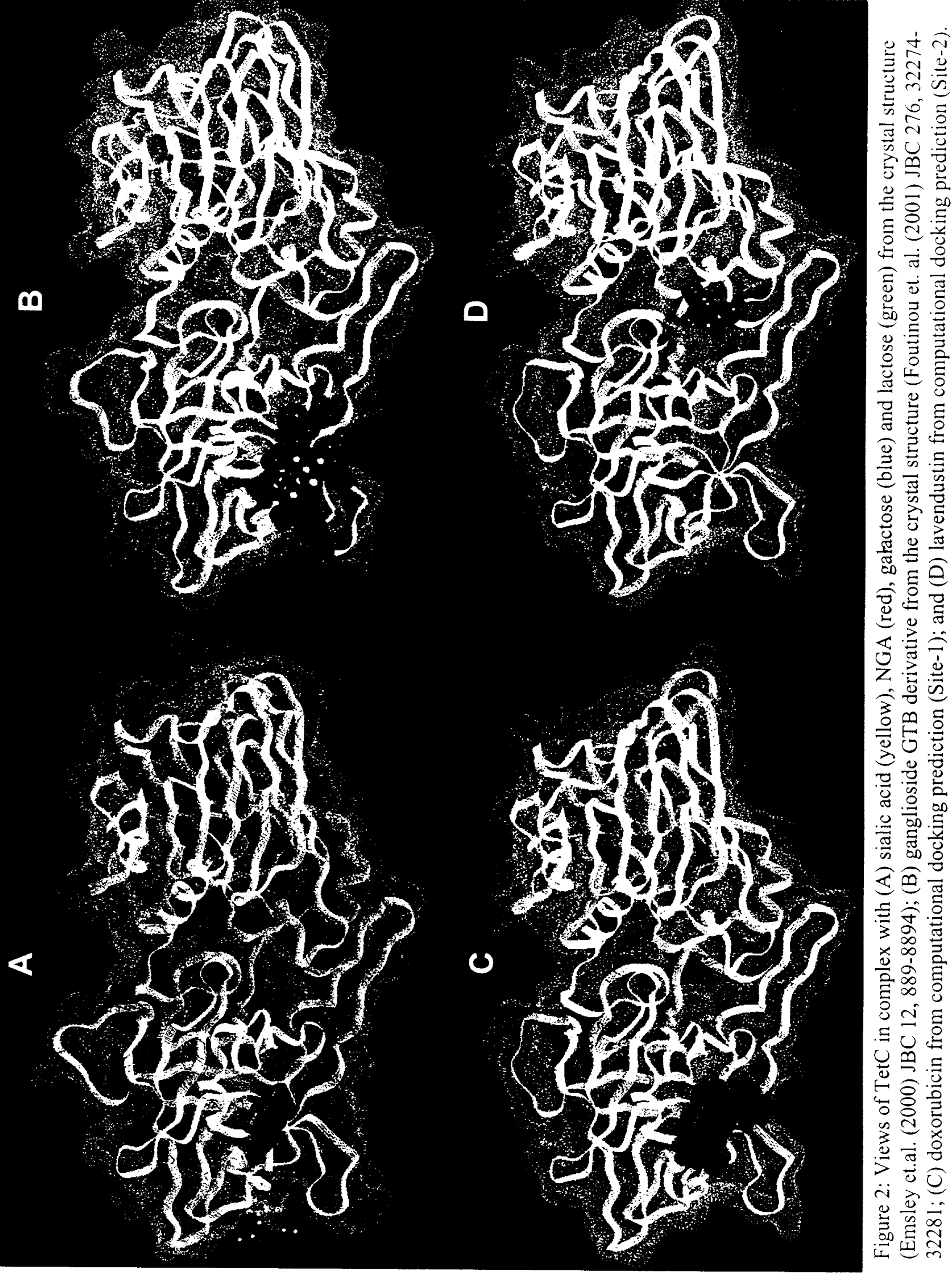



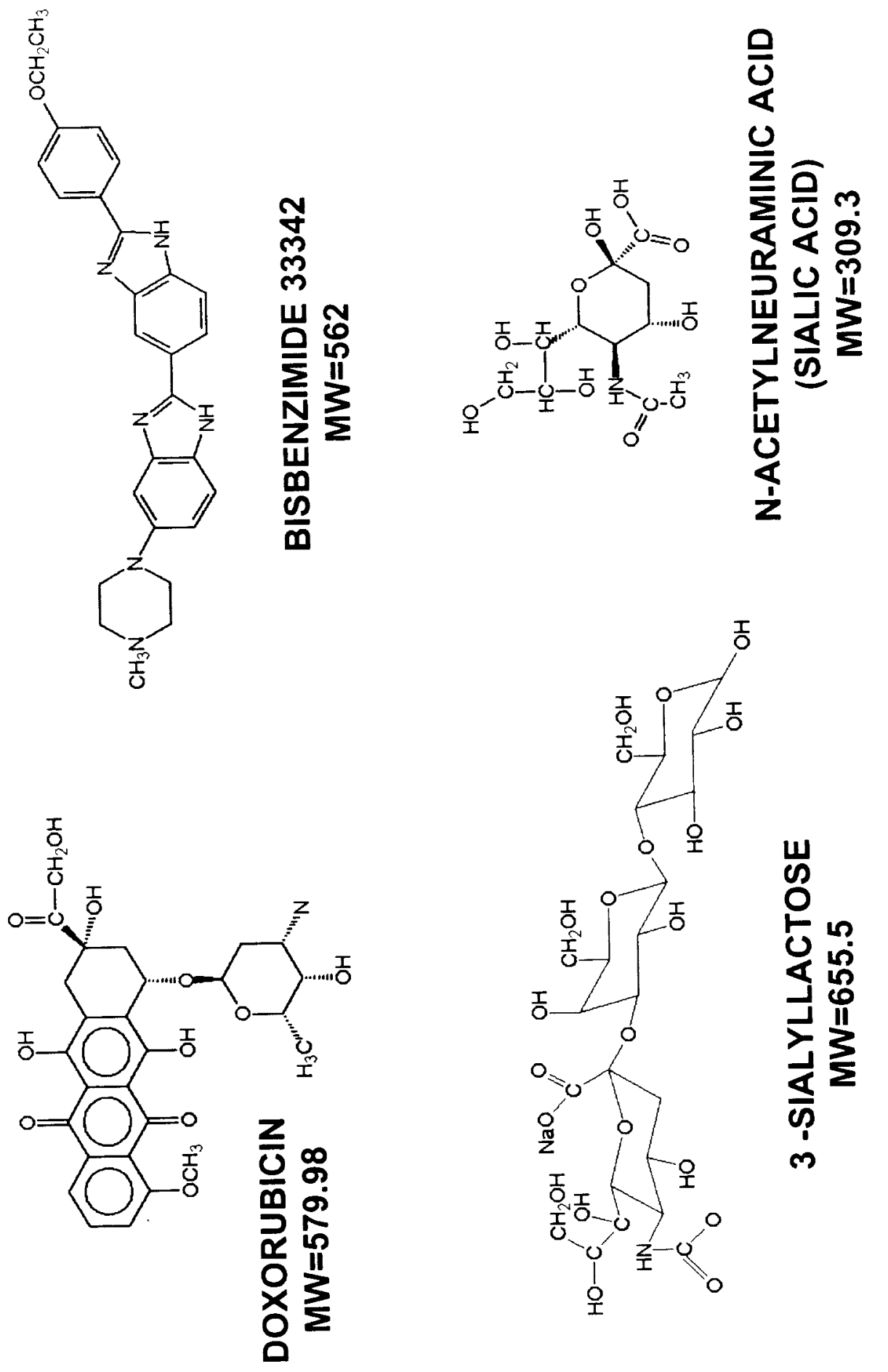

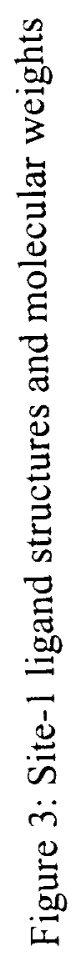




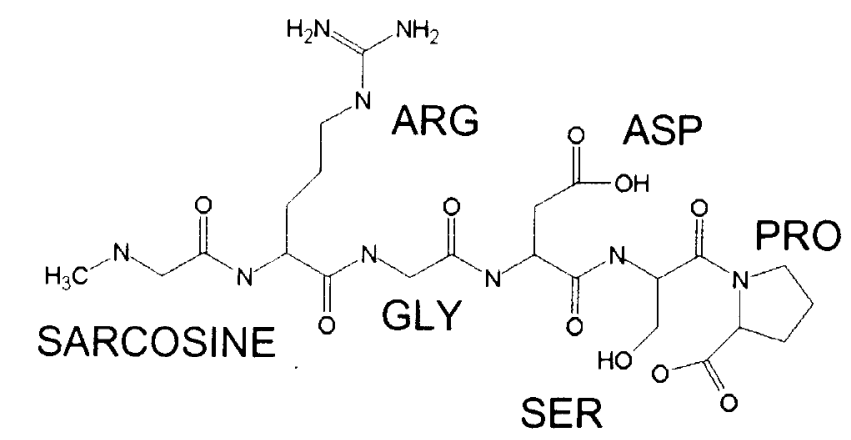

PEPTIDE $M W=601.61$

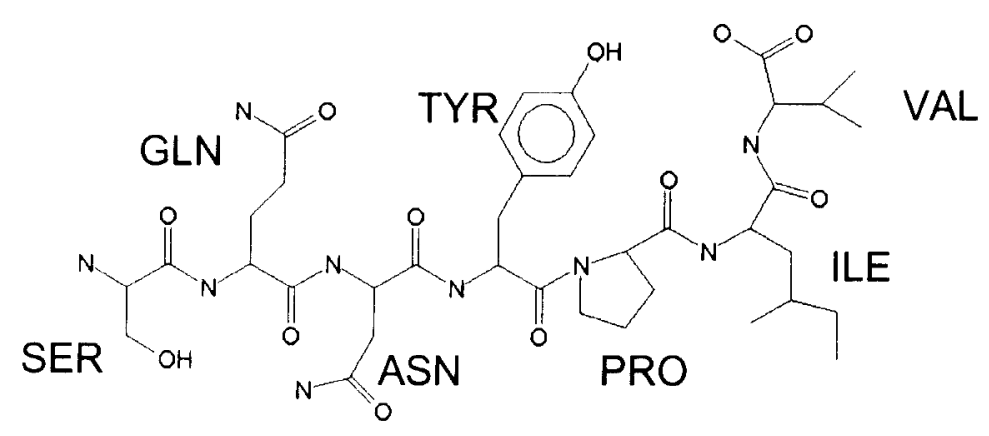

PEPTIDE $M W=819.91$

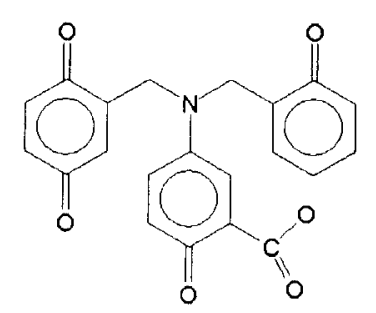

LAVENDUSTIN A $M W=381.38$

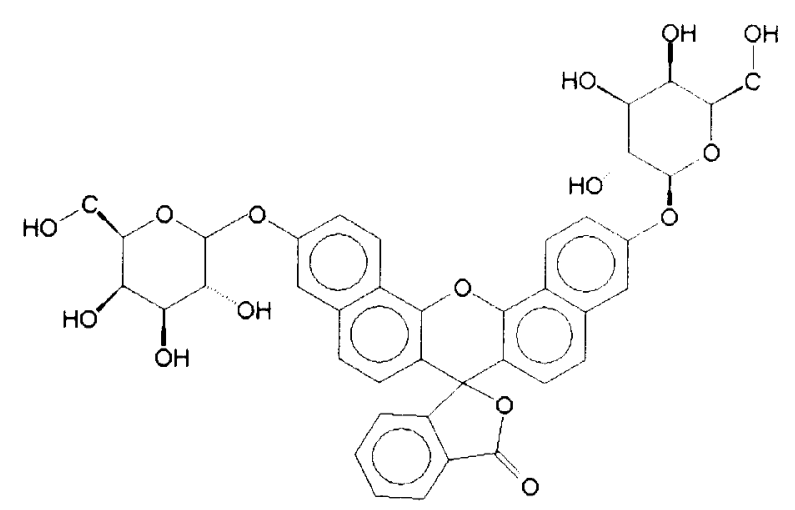

NAPHTHOFLUORESCEIN Di( $\beta$-D GALACTOPYRANOSIDE) $M W=756.71$

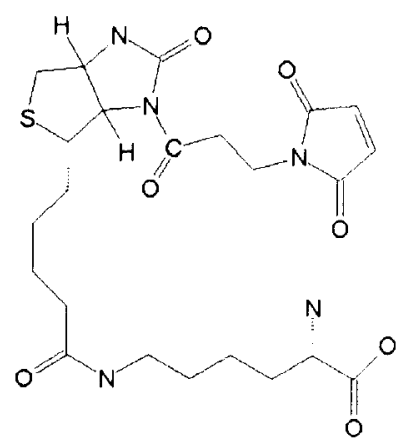

3(N-MALEIMIDOPROPIONYL)BIOCYTIN $M W=523.61$ 


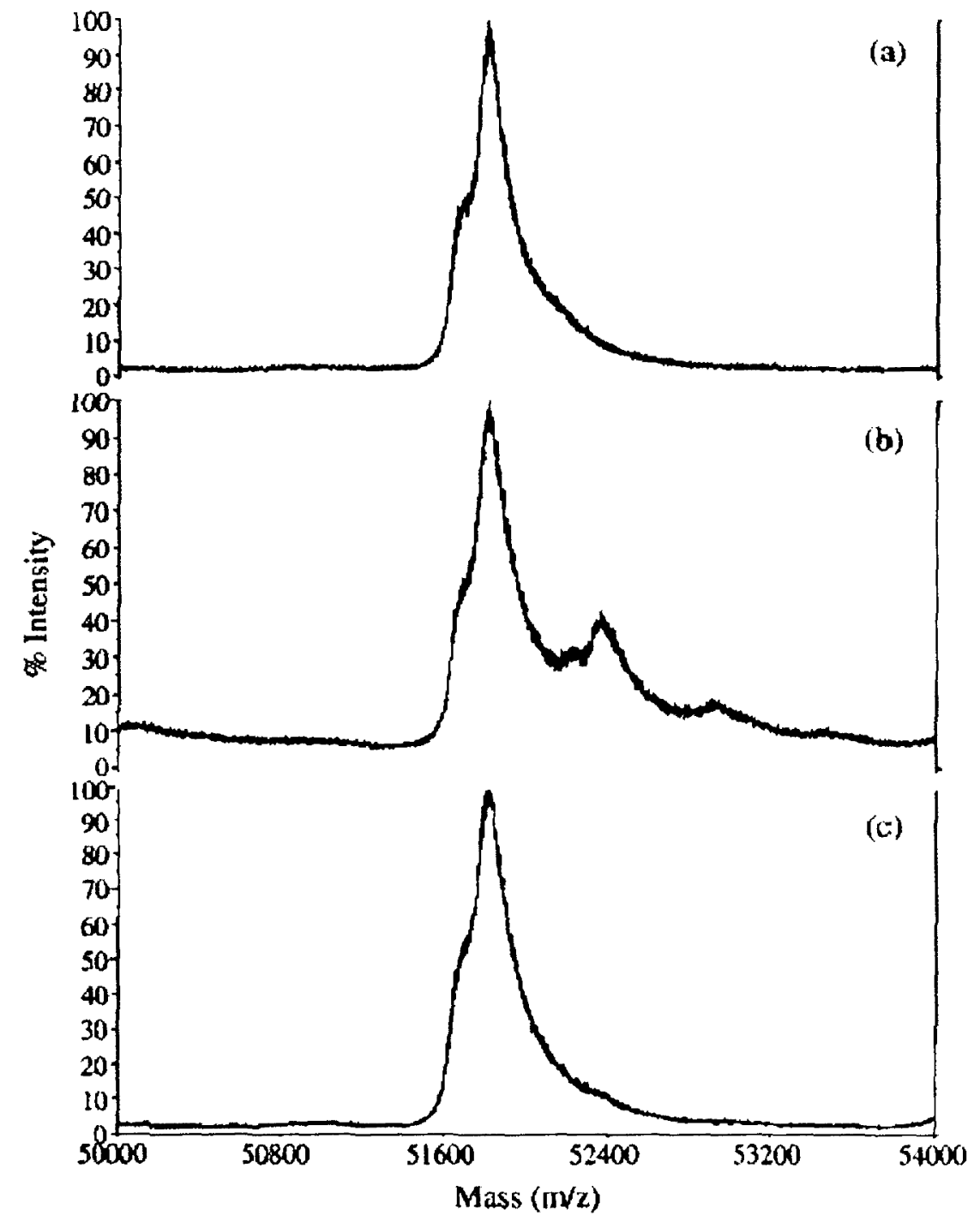

Figure 5: Mass Spectrum of TetC at $100 \mathrm{~V}$. The molecular mass is 51,809.6 Da (b) and (c). The mass spectrum of the complex of lavendustin and $\mathrm{TetC}(\mathrm{b})$ at a declustering voltage of $100 \mathrm{~V}$ showing ligand binding and (c) at a declustering voltage of $200 \mathrm{~V}$ shows no binding. 

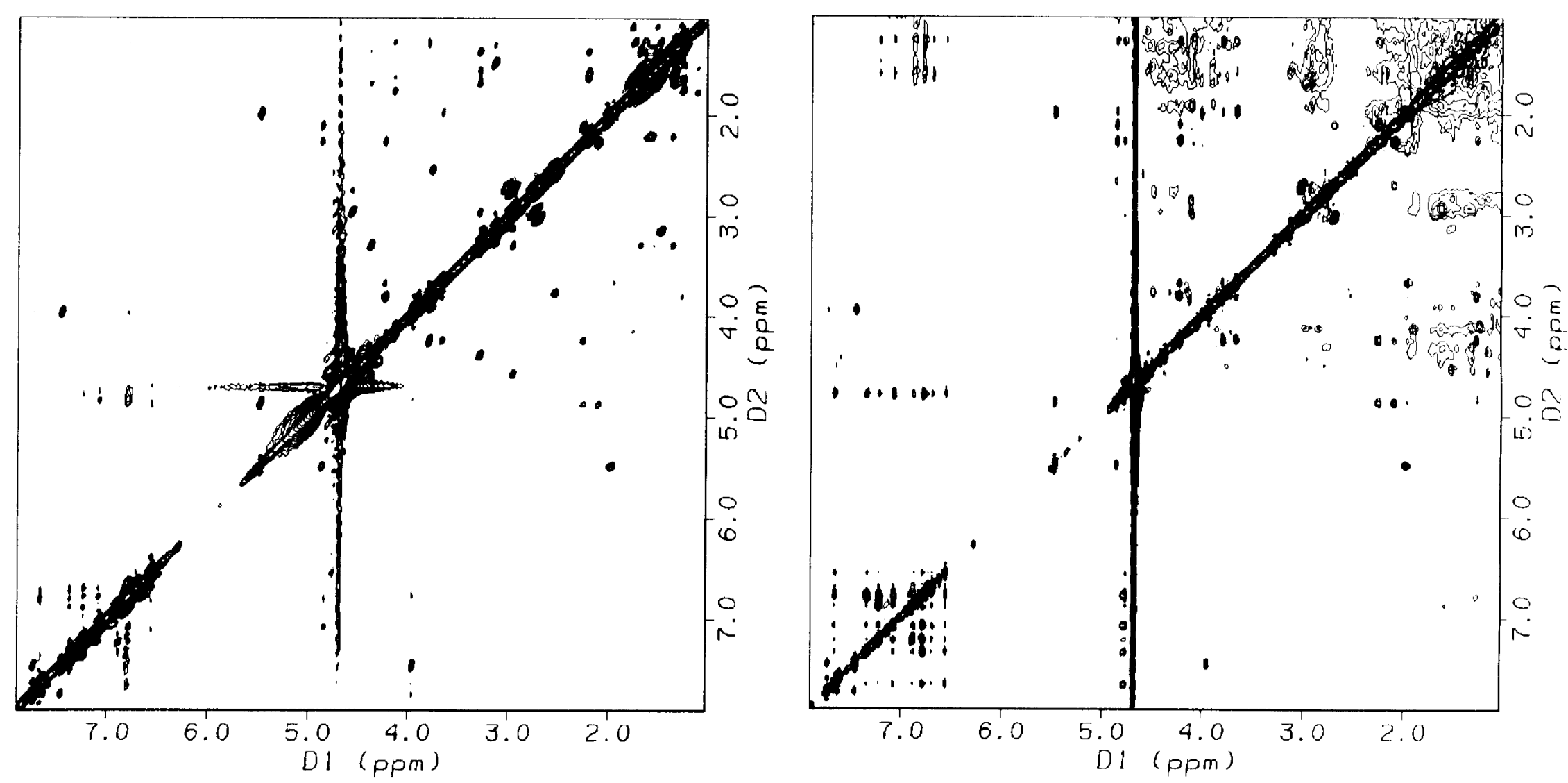

Figure 6: Left: 2D NOESY spectrum at $900 \mathrm{~ms}$ mixing time of the compound mixture composed of doxorubicin, lavendustin, and biocytin in $\mathrm{D}_{2} \mathrm{O}$ at $30^{\circ} \mathrm{C}$. The NOEs are weak and negative for most resonances. Right: 2D transfer NOESY at $300 \mathrm{~ms}$ mixing time of the same mixture in the presence of TetC show strong positive crosspeaks for doxorubicin and lavendustin, indicating that these two ligands bind, while biocytin does not. 


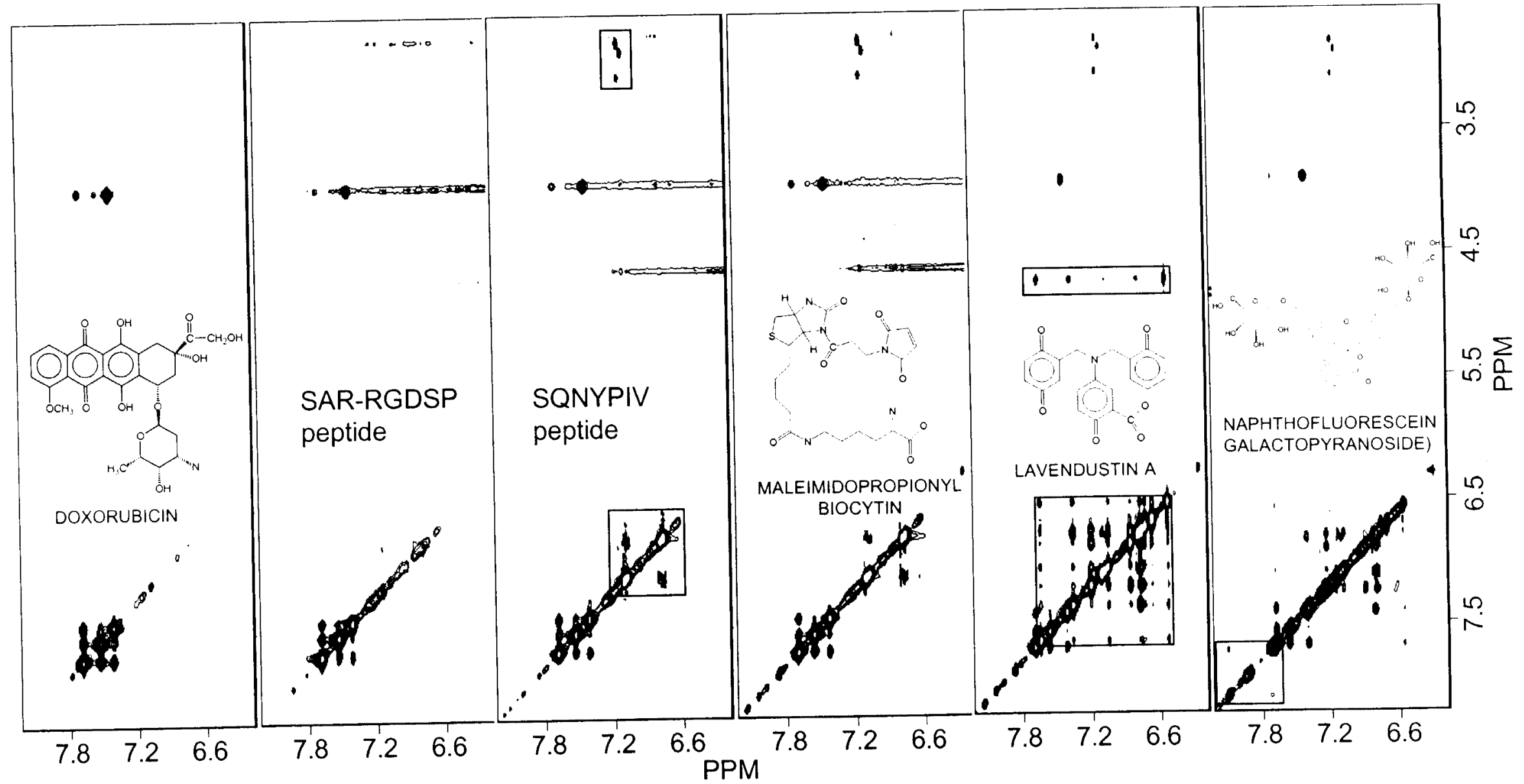

Figure 7: 2D transfer NOESY spectra at $200 \mathrm{~ms}$ mixing time and $20^{\circ} \mathrm{C}$ showing binding of doxorubicin (leftmost panel, Site-1 binder) and a series of predicted Site- 2 binders added sequentially. The presence of new crosspeaks (boxed) indicates binding. Both lavendustin binds and appears to be partially displaced by galactopyranoside, suggesting that these two interact in the same binding site 